\title{
Anogenital Distance in Newborns with Hypospadias
}

\author{
Shashank Patil ${ }^{1}$, Shridhar Ghagane ${ }^{2}$, Manas Sharma ${ }^{1}$, Nerli Rajendra ${ }^{1,}$, , Nitin Pingale ${ }^{3}$, \\ Pulkit Gupta ${ }^{1}$ \\ ${ }^{1}$ Department of Urology, JN Medical College, KLE Academy of Higher Education \& Research, JNMC Campus, Belagavi, India \\ ${ }^{2}$ Urinary Biomarkers Research Centre, Department of Urology, KLES Kidney Foundation, KLES Dr. Prabhakar Kore Hospital \& Medical \\ Research Centre, Nehru Nagar, Belagavi, India \\ ${ }^{3}$ Department of Urology, Bharati Vidyapeeth (Deemed-to-be-University), Pune, Maharashtra, India
}

Email address:

rbnerli@gmail.com (N. Rajendra)

${ }^{*}$ Corresponding author

\section{To cite this article:}

Shashank Patil, Shridhar Ghagane, Manas Sharma, Nerli Rajendra, Nitin Pingale, Pulkit Gupta. Anogenital Distance in Newborns with Hypospadias. American Journal of Pediatrics. Vol. 7, No. 1, 2021, pp. 19-22. doi: 10.11648/j.ajp.20210701.15

Received: September 10, 2020; Accepted: October 5, 2020; Published: March 4, 2021

\begin{abstract}
Hypospadias is a very common congenital anomaly in which the urethra opens onto the ventral aspect of the penis rather than at the tip. It has an incidence of less than $1 \%$ of live male births. In humans, anogenital distance (AGD) is a sexually dimorphic measure of genital development. In this study, we intended to examine the association between AGD and hypospadias using a homogenous group of patients. The same investigator examined all male newborns in our hospital, and anthropometric measurements of the anogenital distance were recorded. All the measurements were made in millimeters $(\mathrm{mm})$ with a sliding digital caliper (graduated in millimeters) used for measuring the distance between the base of the scrotum to the center of the anus; defined as AGD. During the period Jan 2015 to December 2019 (both inclusive), there were 28,426 (14,615 males and 13,811 females) full-term live births in our hospital. The gestational age of the newborns ranged from 37 to 42 weeks. The mean AGD was $21.06 \pm 5.57$ (range 12.11 to 33.14) $\mathrm{mm}$ in newborns without hypospadias, $9.92 \pm 1.90$ (range $8.0-$ 13.9) in newborns with proximal hypospadias and $17.03 \pm 1.95$ (range 14.0 to 19.9 ) $\mathrm{mm}$ with distal hypospadias. In humans, hypospadias is associated with reduced anogenital distance. AGD further decreases with the severity of hypospadias.
\end{abstract}

Keywords: Anogenital Distance, Hypospadias, Newborns

\section{Introduction}

Hypospadias is a very common congenital anomaly, wherein the external urethral opening is situated proximal to the usual glanular location. The defect is thought to origin due to arrested development, though penises in the embryonal phase do not exhibit a similar-appearing stage. [1] In the majority of cases, the aetiology remains unknown. It is well understood that androgens are critical for penile development. The androgen signaling cascade, including testosterone regulation, production, and biosynthesis, the peripheral conversion of testosterone by the enzyme $5-\alpha$ reductase to dihydrotestosterone (DHT), and Testosterone/DHT-androgen receptor interactions mediate virilization of the genital tubercle, resulting in phallic growth and urethral tubularization. [2, 3] It is also clear from the literature that altered androgen signaling, either on a genetic or hormonal level basis, does not entirely account for all human cases of hypospadias and thus is collectively, not solely responsible for hypospadias. [4] Additional molecules are likely to work independently or dependently with androgen signaling, either upstream, downstream, or in concert. Recent investigations into several genetic syndromes have identified additional genes which when mutated or deleted, cause hypospadias, thereby making hypospadias a multifactorial birth defect. [3]

Anogenital distance (AGD), measured from the base of the scrotum (or genital tubercle) to the anus, is a wellknown anthropometric measurement for genital development in animals. $[5,6]$ The United States Environmental Protection Agency has identified it as a sensitive reproductive end-point of masculinization for 
reproductive toxicity studies. [7, 8] AGD is almost twice as long in human males compared with females. This sex difference in AGD is consistent, from 14 to 16-week foetuses, to newborns and infants, and extending until adulthood. [9-11] Anogenital distance has shown to provide a lifelong guide to prenatal androgen exposure during the masculinization programming window of testis development. [12-14] We took up this study to look at the anogenital distance in all the male newborns in our hospital. We compared it with the anogenital distance in the neonates born with hypospadias.

\section{Materials \& Methods}

Study Design: With permission obtained from the Institutional Ethics Committee [KLES/IEC/2014/031], and with the consent of the mother, all male newborns in our hospital were examined by the same investigator. A questionnaire was used to determine the mother's age, parental occupation, mode of delivery, and whether the delivery was single or multiple births. A detailed maternal history, including pre-eclampsia, hypertension, poor weight gain during pregnancy, and medications, was noted.

\section{Approach}

Anthropometric measures of birth weight (in $\mathrm{kg}$ ) and anogenital distance (in $\mathrm{mm}$ ) were recorded. The anogenital measurements were obtained with the newborns lying on their back on a smooth surface with the legs spread and flexed from the knees, by two persons: one holding the baby in place, while the other took the measurement. All the measurements were recorded by the same investigator to minimize inter-investigator variation. Each newborn was placed in the same position, and a single measurement was obtained. This value was recorded as the final measurement. A sliding digital caliper graduated in millimeters was used for the measurements (Figure 1a). In male newborns, the distance between the center of the anus and base of the scrotum was measured (Figure $1 \mathrm{~b}$ ). In female newborns, the distance between the center of the anus and the beginning of the mucosa of the posterior commissure was measured. The male newborn was also examined for hypospadias (Figure 2), undescended testis, hydrocele, and congenital hernia.
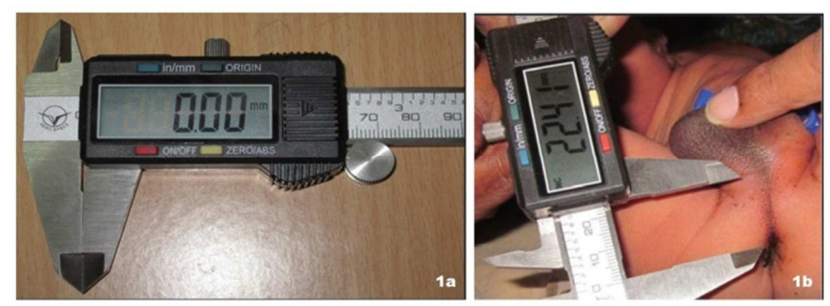

Figure 1. 1a. Digital Vernier caliper instrument. 1b. Measurement of Anogenital distance in a male newborn.

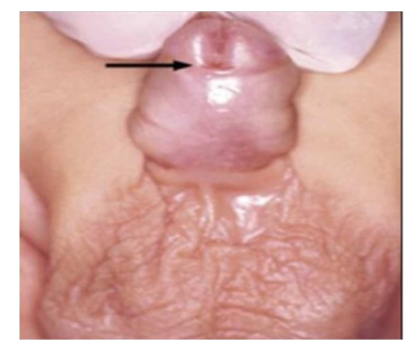

Figure 2. Hypospadias in a newborn.

\section{Results}

During the period Jan 2015 to December 2019 (both inclusive), there were 28,426 (14,615 males and 13,811 females) full-term live births in our hospital (Table 1). The gestational age of the newborns ranged from 37 to 42 weeks. A total of $59(0.4 \%)$ male newborns were diagnosed with hypospadias. The number of males found to have proximal and distal hypospadias was 25 and 34 respectively. The mean AGD was $21.06 \pm 5.57$ (range 12.11 to 33.14 ) $\mathrm{mm}$ in newborns without hypospadias, $9.92 \pm 1.90$ (range $8.0-13.9$ ) in newborns with proximal hypospadias and $17.03 \pm 1.95$ (range 14.0 to 19.9) in newborns with distal hypospadias (Table 2 \& Figure 3 ).

Table 1. Hospital birth records.

\begin{tabular}{|c|c|c|c|c|}
\hline Sl. No & Year & Total live births & Total live Male births & Male with Hypospadias \\
\hline 1 & 2015 & 6324 & 3183 & 14 \\
\hline 2 & 2016 & 6144 & 3079 & 11 \\
\hline 3 & 2017 & 5600 & 3009 & 15 \\
\hline 4 & 2018 & 5383 & 2720 & 9 \\
\hline \multirow[t]{2}{*}{5} & 2019 & 4975 & 2624 & 10 \\
\hline & TOTAL & 28426 & $14615(51.41 \%)$ & $59(0.40 \%)$ \\
\hline
\end{tabular}

Table 2. Anogenital distance (mean $\mathrm{mm}$ ).

\begin{tabular}{llll}
\hline & $\begin{array}{l}\text { Mean AGD - Male Newborns without } \\
\text { hypospadias }\end{array}$ & $\begin{array}{l}\text { Mean AGD - with proximal } \\
\text { hypospadias (n 25) }\end{array}$ & $\begin{array}{l}\text { Mean AGD - with distal } \\
\text { hypospadias (n 34) }\end{array}$ \\
\hline 1 & & $9.92 \pm 1.90$ & $17.03 \pm 1.95$ \\
2 & $21.06 \pm 5.57$ & $13.95 \pm 4.03$ & $<0.0001$ \\
\hline
\end{tabular}

The mean AGD of Newborns without hypospadias was significantly longer than the AGD of Newborns with hypospadias ( $<<0.01$ ).

Similarly, the AGD of Newborns with distal hypospadias was significantly longer than AGD of Newborns with proximal hypospadias ( $<<0.01$ ). 


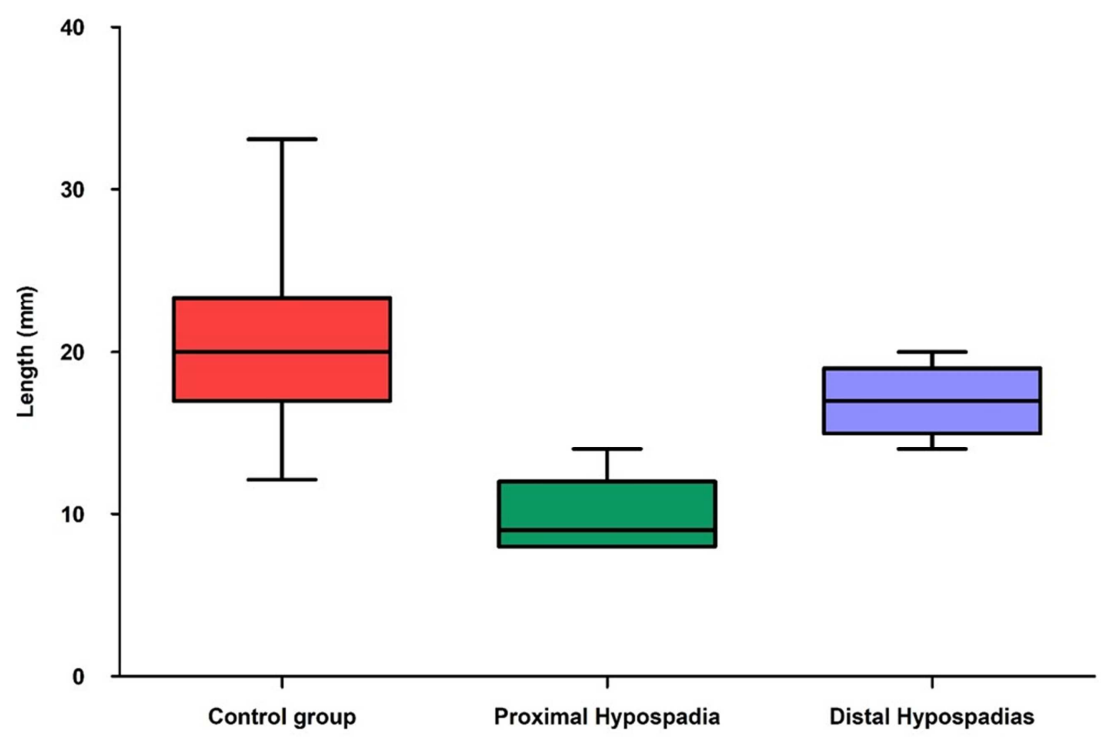

Figure 3. Anogenital distance as per the severity of hypospadias.

\section{Discussion}

Measurements of anogenital distances have been reported in several previous studies. [15-17] The accuracy of these measurements can be affected by the apparatus used for the measurements, by the number of measurements and by the experience of the person taking the measurements, as well as by the number of investigators involved in recording the measurement. [18] Flexible tape measurements can increase the margin of error. However, Hsieh et al. [19] reported that accuracy could be improved by using a flexible ruler that conformed to the natural curve of the perineum and measured under anaesthesia. Adjustable Vernier calipers, which is a rigid apparatus, helps to improve accuracy. We have similarly used digital Vernier calipers in our study to assist us in improving our measuring accuracy.

Previous human studies have used different protocols to measure AGD. Salazar-Martinez et al. [17] measured AGD from the base of the scrotum in males or the posterior fourchette in females to the center of the anus. Others have measured AGD in males from the anterior and posterior base of the penis to the anus, similar to the measurement used in rodents. [19] In the present study, we chose the former protocol because the anatomical landmarks are easily recognizable. The anterior anatomical landmark in our study indicates the location of the caudal border of the genital swelling, the Anlagen that differentiates as the labia majora in females and the scrotum in males.

Perineal growth and caudal migration of the genital tubercle are androgen-dependent processes in male rodents. [21] Measurement of AGD is considered to be a sensitive index of prenatal androgen exposure. Hence, it could be used in humans as a marker of the antiandrogens effects of various endocrine-disrupting chemicals (EDCs). The external genitalia in humans is sexually dimorphic at birth, with the genital tubercle differentiated into a penis or clitoris. The purported mechanism by which androgens increase AGD is by inducing labio-scrotal fusion, which begins caudally and proceeds ventrally.

In children, a shorter anogenital distance has been linked to cryptorchidism, hypospadias, and micropenis. However, there is limited data in the literature regarding anogenital distance measurements in the foetuses. Gilboa et al. [20] prospectively measured anogenital in all cases referred for suspected isolated abnormal male genitalia and compared it with anogenital distance prenatal measurements. All foetuses with hypospadias had an anogenital distance measurement below the fifth percentile. Statistical analysis showed a significant difference between the average mean anogenital distance for gestational age versus those with hypospadias $(\mathrm{Mean} \pm \mathrm{SD}, 16.90 \pm 4.08$ and $11.68 \pm 3.31 \mathrm{~mm}$, respectively; $\mathrm{p}=0.001$ ). The authors concluded that foetuses with hypospadias had a statistically significant shorter anogenital distance compared with the general population, and that, the anogenital distance could serve as a complementary objective sonographic parameter in the prenatal assessment and counseling of male external genital anomalies.

Hua et al. [21] conducted a meta - analysis to assess gradations in the severity of the endocrine disruption in cryptorchidism or hypospadias by using AGD. A total of 2,119 boys from five birth cohort studies and two cross sectional studies were subjected to meta - analysis. A random-effect model was used to calculate the standardized mean difference (SMD) of AGD. Their results revealed that boys with hypospadias or cryptorchidism had a shorter AGD. Cox et al. [22] evaluated the relationship between the severity of hypospadias and AGD in 59 boys with hypospadias and 31 age-matched controls undergoing circumcision. They reported that the more severe forms of hypospadias were associated with shorter AGD and ASD (anoscrotal distance). Similarly, Hsieh et al. [23] measured the AGD in boys with hypospadias and those with normal genitals. In their study, AGD measurement was done in 119 
Caucasian boys with age ranging from 4 to 86 months; 42 boys had normal genitalia, and 77 were found to have hypospadias. The mean $( \pm \mathrm{SD})$ AGD of boys with hypospadias was $67 \pm 1.2$ versus $73 \pm 1 \mathrm{~mm}$ for boys with normal genitals $(p=0.002)$. After matching for age (all $<2$ years of age), boys with hypospadias $(n=26)$ still featured a shorter AGD than boys with normal genitals $(n=26 ; 62 \pm 2$ versus $68 \pm 2 \mathrm{~mm}$ respectively, $\mathrm{p}=0.033$ ) but the differences in age, height and weight were no longer significant.

\section{Conclusion}

Our study supports an association between hypospadias and anogenital distance. A shortened anogenital distance in the setting of hypospadias may be a sign of global phenotypic changes in under-virilized boys. Anogenital distance further decreases with the severity of hypospadias. In humans, hypospadias is associated with reduced anogenital distance.

\section{Conflict of Interest}

The authors declare conflict of interest as None.

\section{References}

[1] Snodgrass WT \& Bush NC. Hypospadias. In Wein AJ, Kavoussi LR, Partin AW \& Peters CA Ed. Campbell-Walsh Urology, 11 ${ }^{\text {th }}$ ed., Elsevier, Philadelphia. 2016 p 3399.

[2] Baskin LS. Hypospadias and urethral development. J Urol. 2000; 163: 951-56.

[3] Yucel S, Dravis C, Garcia N, Henkemeyer M, Baker LA. Hypospadias and anorectal malformations mediated by defective Eph/ephrin signaling. Journal of pediatric urology. 2007; 3 (5): 354-63.

[4] Holmes N, Miller W, Baskin L. Lack of defects in androgen production in children with hypospadias. J Clin Endocrinol Metab 2004; 89: 2811-2816.

[5] McIntyre BS, Barlow NJ, Foster PM. Androgen-mediated development in male rat offspring exposed to flutamide in utero: permanence and correlation of early postnatal changes in anogenital distance and nipple retention with malformations in androgen-dependent tissues. Toxicol Sci 2001; 62: 236-249.

[6] Bowman CJ, Barlow NJ, Turner KJ, Wallace DJ, Foster PM. Effects of in utero exposure to finasteride on androgendependent reproductive development in the male rat. Toxicol Sci 2003; 74: 393-406.

[7] Nerli RB, Ghagane SC, Hiremath MB, Dixit NS, Neelagund S Anogenital distance in males attending assisted reproduction center. Journal of the Scientific Society. 2018; 45 (2): 72.

[8] Gore AC, Chappell VA, Fenton SE, Flaws JA, Nadal A, Prins GS, Toppari J, Zoeller RT. EDC-2: The Endocrine Society's Second Scientific Statement on Endocrine-Disrupting Chemicals. Endocr Rev 2015; 36: E1-E150.
[9] Papadopoulou E, Vafeiadi M, Agramunt S, et al. Anogenital distances in newborns and children from Spain and Greece: predictors, tracking and reliability. Paediatr Perinat Epidemiol 2013; 27: 89-99.

[10] Sathyanarayana S, Grady R, Redmon JB, et al. Anogenital distance and penile width measurements in The Infant Development and the Environment Study (TIDES): methods and predictors. J Pediatr Urol 2015; 11: 76 e71-76.

[11] Fowler PA, Filis P, Bhattacharya S, et al. Human anogenital distance: an update on fetal smoke-exposure and integration of the perinatal literature on sex differences. Hum Reprod 2016; 31: 463-472.

[12] Welsh M, Saunders PT, Fisken M, Scott HM, et al. Identification in rats of a programming window for reproductive tract masculinization, disruption of which leads to hypospadias and cryptorchidism. J Clin Invest 2008; 118: $1479-1490$

[13] Macleod DJ, Sharpe RM, Welsh M, et al. Androgen action in the masculinization programming window and development of male reproductive organs. Int J Androl 2010; 33: 279-287.

[14] van den Driesche S, Scott HM, MacLeod DJ, Fisken M, Walker M, Sharpe RM. Relative importance of prenatal and postnatal androgen action in determining growth of the penis and anogenital distance in the rat before, during and after puberty. Int J Androl 2011; 34: e578-e586.

[15] Thankamony A, Ong KK, Dunger DB, et al. Anogenital distance from birth to 2 years: a population study. Environ Health Perspect. 2009; 117: 1786-90.

[16] Orish C, Didia B. Anogenital distance in human male and female newborns: A look at a cross section of a Nigerian population. Internet J Biol Anthropol. 2008; 3 (2): e1-4.

[17] Salazar-Martinez E, Romano-Riquer P, Yanez-Marquez E, et al. Anogenital distance in human male and female newborns: A descriptive, cross-sectional study. Environ Health 2004; 3: 8.

[18] Ozkan B, Konak B, Cayir A, et al. Anogenital Distance in Turkish Newborns. J Clin Res Ped Endo 2011; 3 (3): 122-125.

[19] Hsieh MH, Breyer BN, Eisenberg ML, Baskin LS (2008) Associations among hypospadias, cryptorchidism, anogenital distance, and endocrine disruption. Curr Urol 2008; 9: 137-42.

[20] Gilboa Y, Perlman S, Kivilevitch Z, et al. Prenatal Anogenital Distance Is Shorter in Fetuses with Hypospadias. J Ultrasound Med 2017; 36: 175-182.

[21] Hua XG, Hu R, Hu CY, et al. Associations between hypospadias, cryptorchidism and anogenital distance: systematic review and meta-analysis. Andrologia 2018; 50 (10): e 13152.

[22] Cox K, Kyriakou A, Amjad B et al. Shorter anogenital and anoscrotal distances correlate with the severity of hypospadias: a prospective study. J Paed Urol 2017; 13: 57. e1-57. e5.

[23] Hsieh MH, Eisenberg ML, Hittelman AB, et al. Caucasian male infants and boys with hypospadias exhibit reduced anogenital distance. Hum Reprod 2012; 27: 1577-1580. 